\title{
Fault Tolerance for Industrial Actuators in Absence of Accurate Models and Hardware Redundancy
}

Papageorgiou, Dimitrios; Blanke, Mogens; Niemann, Hans Henrik; Richter, Jan H.

\section{Published in:}

Proceedings of the 2015 IEEE Multi-Conference on Systems and Control (MSC)

Link to article, DOI:

10.1109/CCA.2015.7320885

Publication date:

2015

Document Version

Peer reviewed version

Link back to DTU Orbit

Citation (APA):

Papageorgiou, D., Blanke, M., Niemann, H. H., \& Richter, J. H. (2015). Fault Tolerance for Industrial Actuators in Absence of Accurate Models and Hardware Redundancy. In Proceedings of the 2015 IEEE Multi-Conference on Systems and Control (MSC) (pp. 1887-1894). IEEE. https://doi.org/10.1109/CCA.2015.7320885

\section{General rights}

Copyright and moral rights for the publications made accessible in the public portal are retained by the authors and/or other copyright owners and it is a condition of accessing publications that users recognise and abide by the legal requirements associated with these rights.

- Users may download and print one copy of any publication from the public portal for the purpose of private study or research.

- You may not further distribute the material or use it for any profit-making activity or commercial gain

- You may freely distribute the URL identifying the publication in the public portal 


\title{
Fault Tolerance for Industrial Actuators in Absence of Accurate Models and Hardware Redundancy
}

\author{
Dimitrios Papageorgiou ${ }^{1}$, Mogens Blanke ${ }^{1,2}$, Hans Henrik Niemann ${ }^{1}$ and Jan H. Richter ${ }^{3}$
}

\begin{abstract}
This paper investigates Fault-Tolerant Control for closed-loop systems where only coarse models are available and there is lack of actuator and sensor redundancies. The problem is approached in the form of a typical servomotor in closed-loop. A linear model is extracted from input/output data to describe the system over a frequency range. Two methods based on the Kalman Filter and Statistical Change Detection techniques are proposed for detecting degradation faults and component failures, respectively. Finally, a reference correction setup is used to compensate for degradation faults.
\end{abstract}

\section{INTRODUCTION}

Maximum system availability and optimal performance are quintessential in all modern automated processes. As a consequence, fault-tolerant operation is highly desirable in technologically complex tasks, especially in industrial applications, where system failures often lead to costly situations, such as unplanned production stops, or even worse, in situations where safety of persons is compromised.

Most existing methods for Fault Detection and Isolation (FDI) are model-based, both with respect to the techniques used for detecting faults and to fault accommodation. An approach to FDI for linear time-invariant (LTI) systems with no prior model knowledge was presented in [1], where only the structural characteristics of the system and the faults were assumed to be known. Results from Distribution Theory were used to estimate selected fault characteristics, the time evolution of which formed the basis for a decision policy (to decide whether a fault appears to be present). A different approach was pursued in [2] where Active Fault Detection and Isolation (AFDI) was applied in uncertain systems and in [3], where normal changes in use-modes of a system were exploited to isolate faults.

The approach suggested in this paper to the general Fault-Tolerant Control (FTC) problem is based on utilising behavioural characteristics of a given system to obtain a basic model. This model is generic, and the unknown parameters are estimated using off-line System Identification techniques. Results from model-based FDI methods employing Kalman filters are then used for diagnosis. As a further step, a method for FDI and FTC is developed that circumvents the necessity of detailed models and that do not require direct access to the

\footnotetext{
${ }^{1}$ Department of Electrical Engineering, Automation and Control, Technical University of Denmark, DK-2800 Kgs. Lyngby, Denmark dimpa, mb, hhn@elektro.dtu.dk

${ }^{2}$ AMOS CoE, Institute of Technical Cybernetics, Norwegian University of Science and Technology, Trondheim, Norway

${ }^{3}$ Siemens AG, Process Industries and Drives, D-90475 Nuremberg, Germany janrichterdsiemens.com
}

control signal. This new approach could make fault-tolerance an "easy-to-obtain" feature for existing industrial plants.

This study considers a system of a servomotor in closedloop and addresses FTC possibilities under the limitations that prevail in industrial manufacturing. These limitations include the lack of information about the system dynamics, structure and parameters, and the inability to easily changing the control signal internally in the control cascade. The industrial relevance is emphasised by avoiding assumptions on supplemental instrumentation that would not be present in a standard factory floor automation.

This paper investigates the envelope of fault accommodation possibilities under degradation faults and suggests generic compensation solutions using a reference correction setup. The contribution is a novel technique to detect component failures, that can be applied on a generic singleinput single-output (SISO) system when the only available information is the input/output behaviour of the system. This problem is treated theoretically in the first six sections, where methods for extracting a simple model are discussed and suitable FDI and FTC techniques are introduced. Finally, the last two sections demonstrate the methods applied in a simulation environment and conclusions are drawn.

\section{PROBLEM FORMULATION}

A the test-bed system is described in this section focusing on equipment and available information. Generic faults are presented, the FTC objectives are stated and basic assumptions are made which are needed in the later analysis.

\section{A. System description}

The system consists of a typical servomotor controlled by a standard PID controller with known parameters. Attached to the motor shaft is there a potentiometer for measuring the angular position of the motor. This is the only sensor available. Although in typical industrial systems there are more complex dynamics, under certain assumptions these dynamics can be decoupled. A characteristic example is the industrial Cartesian robots ([4]). Moreover, in articulated robotic manipulators, when the gearing between the joint motors is reducing the coupling torques between the robot links, or when the torque developed due to the weight of the target object is insignificant, the principle of Independent Joint Control can be adopted ([5] and [6]). Then, each motor can be studied separately and, as such, the FDI analysis can be reduced to that of each single servomotor separately. 


\section{B. Considered faults}

Two classes of faults are considered in this study:

- degradation faults

- complete failures of components

Degradation faults do not compromise the entire functionality of the piece of equipment they appear in. However, they affect its performance and, as a consequence, of the system itself. They can be modelled as additive signals in the dynamics or the output equations of the system (e.g. corrupted sensor readings).

On the other hand, failed components are rendered completely unusable and, as such, failures of this type correspond to complete loss of pieces of equipment. An example of a failed component is a broken motor (actuator failure).

\section{Assumptions}

A number of assumptions illustrating the limitations found in many industrial applications are made, so as to create the framework of the analysis.

Specifically, it has been assumed that the only sensor available is the motor position sensor. Regarding the knowledge about the structure of the system, only the nominal controller and a series of $\mathrm{I} / \mathrm{O}$ data (reference to angular position tuples) are available. Finally, there is no possibility of changing the control signal (adding signals to it) or the controller itself in the servomotor (the internal control loop cannot be broken).

After the framework of the system and the relevant assumptions have been presented, the problem this paper addresses can now be stated:

Problem 1. Under the assumptions presented in section IIC design a FTC setup for the closed-loop servomotor system.

\section{BLACK-BOX FTC}

Since by assumption the only access available to the system is the reference commands and the output readings, any analysis of the system is limited to inspection of the closed-loop behaviour of the system. This implies that the possibilities for FTC must be based on monitoring and remedying any discrepancies between the real system and its closed-loop model. The approach can be referred to as Black-box Fault-tolerant Control.

Given that the servomotor is a linear SISO system with not identically zero transfer function, any change in the closedloop behaviour can be expressed as the effect of variation of the reference signal and will be perceived as a variation of the nominal output signal. This motivates the idea of detecting faults by monitoring the $\mathrm{I} / \mathrm{O}$ pairs of the system and describe the detected faults as variations of the nominal reference as illustrated in Figure 1.

If the signal $\delta$, which is the appropriate reference variation that would cause the nominal system to respond with the faulty output $y_{f}$ (the same with the response of the faulty system), is accurately estimated, then it can be subtracted from the nominal reference, so that the fault can be compensated for.

Since no hardware redundancies are available, only degradation faults can be compensated for through appropriate

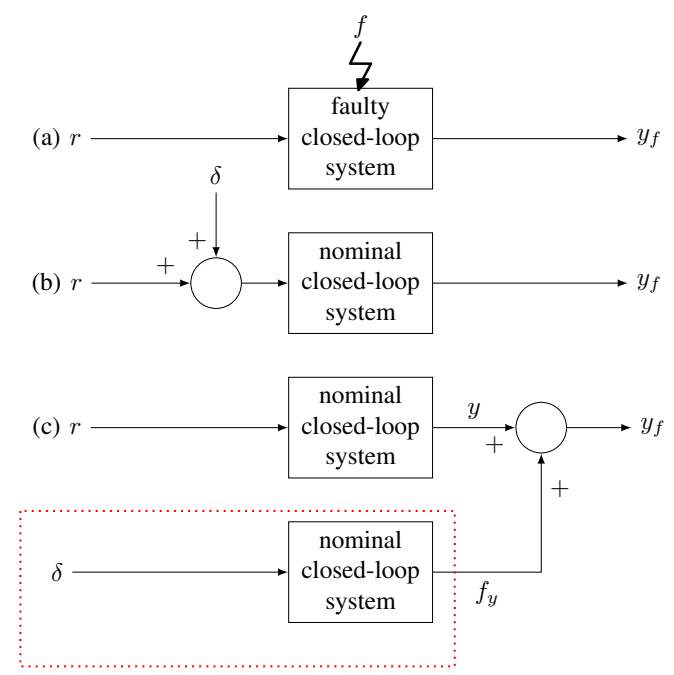

Fig. 1: Faulty system equivalent descriptions: (a) Plant fault description. (b) Input fault description. (c) Output fault description.

modification of the nominal reference, whereas in case of component failures, the system must be shut down. However, it is possible to detect complete failures, as it will be shown in Section VI. This is due to the fact that the description of an actuator or sensor failure as a reference variation takes the form of very specific measurable signals that cannot be compensated. Hence, failure detection reduces into using statistical change detection techniques to examine if the estimated reference perturbation matches the dynamical profile of these signals.

The approach relies on having a description of the nominal closed-loop system, which by assumption is not available in advance. As such, a linear model of the closed-loop system has to be identified before any of the techniques can be applied. This identification is done once, offline and based on fault-free reference-to-output pairs. Overall, the developments needed for a complete FTC setup consistent to the assumptions made can be summarized in the following:

- Obtain a linear model for the nominal closed-loop system through offline System Identification

- Account for model uncertainties

- Design an estimator for the reference variation $\delta$

- Estimate $\delta$ and correct the reference

- Compare $\delta$ in the compensated system to known dynamical profiles for failure detection

\section{Closed-LOOP SYSTEM DYNAMICS}

\section{A. Motor dynamics}

Throughout this analysis the dynamics of the system are considered to lie at relatively low frequencies. This is often the case in many industrial applications with robots performing typical pick-and-place tasks. As such, a linear $2^{\text {nd }}$ order system is a good approximation of the closedloop dynamics of the motor (see more on frequency-weighted model reduction in [7] and [8]). If $G(s)$ is the stable, proper closed-loop transfer function of the servomotor, where the 
denominator is a monic polynomial as below

$$
G(s)=\frac{b_{1} s+b_{0}}{s^{2}+a_{1} s+a_{0}}
$$

then four parameters must be estimated to obtain a full description of the closed-loop motor system.

Through a grey-box identification method based on the minimization of the parameter prediction error ([9]), the approximated model parameters can be calculated offline. This identification concerns only the nominal closed-loop system. As such, faults are not included in the identification algorithm.

\section{B. Uncertain model}

The approximation of the motor dynamics with a lower order model, as well as the parameter identification method itself, introduce a degree of uncertainty into the proposed model. This uncertainty can be modelled as additive deviations in the transfer function, the input or the output of the system. This approach has been motivated by the following proposition.

Proposition 1: For a Linear Time-Invariant (LTI) system with not identically zero transfer function, any parameter perturbation can be expressed as a time-varying perturbation of the nominal output signal.

Proof: Let

$$
\Sigma_{N}:\left\{\begin{array}{l}
\dot{x}(t)=A x(t)+B u(t) \\
y(t)=C x(t)
\end{array} \quad, x\left(t_{0}\right)=x_{0}\right.
$$

be a realisation of an $n^{\text {th }}$-order LTI system, where $x \in \mathbb{R}^{n}$, $u \in \mathbb{R}^{m}, y \in \mathbb{R}^{p}, A \in \mathbb{M}^{n \times n}, B \in \mathbb{M}^{n \times m}$ and $C \in \mathbb{M}^{p \times n}$. Then the general solution $x(t)$ is given by

$$
x(t)=e^{A\left(t-t_{0}\right)} x_{0}+\int_{t_{0}}^{t} e^{A(t-\tau)} B u(\tau) d \tau
$$

while the system nominal output is

$$
y(t)=y_{n o m}(t)=C e^{A\left(t-t_{0}\right)} x_{0}+\int_{t_{0}}^{t} C e^{A(t-\tau)} B u(\tau) d \tau
$$

Moreover, let

$$
\Sigma_{N_{\Delta}}:\left\{\begin{array}{l}
\dot{x}(t)=(A+\delta A) x(t)+(B+\delta B) u(t) \\
y_{\Delta}(t)=(C+\delta C) x(t)
\end{array}\right.
$$

be a variation of the nominal system $\Sigma_{N}$. Define the matrix function $\psi_{n}: \mathbb{R} \rightarrow \mathbb{M}^{n \times n}$ as

$$
\psi_{n}(t)=e^{(A+\delta A) t}-e^{A t}
$$

Following the result from equation (2) the solution of the perturbed system is given by:

$$
\begin{aligned}
x_{\Delta}(t) & =e^{(A+\delta A)\left(t-t_{0}\right)} x_{0}+ \\
& +\int_{t_{0}}^{t} e^{(A+\delta A)(t-\tau)}(B+\delta B) u(\tau) d \tau=x(t)+\delta_{1}(t)
\end{aligned}
$$

where

$$
\begin{aligned}
\delta_{1}(t) & =\psi_{n}\left(t-t_{0}\right) x_{0}+\int_{t_{0}}^{t} e^{A(t-\tau)} \delta B u(\tau) d \tau+ \\
& +\int_{t_{0}}^{t} \psi_{n}(t-\tau)(B+\delta B) u(\tau) d \tau
\end{aligned}
$$

The output of the perturbed system is, therefore, written as

$$
\begin{aligned}
y_{\Delta}(t) & =(C+\delta C) x(t)=C x(t)+\delta C x(t)= \\
& =y_{n o m}(t)+\delta(t)
\end{aligned}
$$

where

$\delta(t)=\delta C e^{A\left(t-t_{0}\right)} x_{0}+\int_{t_{0}}^{t} \delta C e^{A(t-\tau)} B u(\tau) d \tau+(c+\delta C) \delta_{1}(t)$

Thus, the output of the perturbed system is a time-varying perturbation of the nominal output.

Proposition 1 shows that the uncertainties can be modelled as additive signals in the output of the system. The next proposition extends this consideration for any bounded variation $\delta(t)$ of the output, being expressed as an input perturbation.

Proposition 2: For a Linear Time-Invariant (LTI) system with not identically zero transfer function, any output perturbation can be expressed as the effect of a time-varying perturbation of the nominal input signal.

Proof: Consider the nominal system $\Sigma_{N}$ and its variation $\Sigma_{N_{\Delta}}$ from Proposition 1 with a more generic perturbation of the output $y_{\Delta}(t)=y(t)+\delta(t)$, where $\delta(t)$ is piecewise continuously differentiable. The output of the perturbed system can be written as

$$
\begin{aligned}
y_{\Delta}(t) & =C x(t)+\delta(t)=C\left[x(t)+C^{+} \delta(t)\right]= \\
& =C\left[e^{A\left(t-t_{0}\right)} x_{0}+\int_{t_{0}}^{t} e^{A(t-\tau)} B u(\tau) d \tau+C^{+} \delta(t)\right]
\end{aligned}
$$

where $C^{+}$denotes the pseudoinverse of matrix $C$.

Proving that $\delta(t)$ can be expressed as a variation of the input signal $u(t)$ is equivalent to proving that $\exists \delta u(t) \in \mathbb{R}^{m}$ :

$$
C^{+} \delta(t)=\int_{t_{0}}^{t} e^{A(t-\tau)} B \delta u(\tau) d \tau
$$

Differentiating both parts of Equation (5) and taking $\delta u\left(t_{0}\right)=0$ yields

$$
\begin{array}{r}
{\left[e^{A(t-\tau)} B \delta u(\tau) d \tau\right]_{t_{0}}^{t}=C^{+} \dot{\delta}(t) \Leftrightarrow} \\
B \delta u(t)=C^{+} \dot{\delta}(t) \Leftrightarrow \\
\delta u(t)=B^{+} C^{+} \dot{\delta}(t)
\end{array}
$$

where $B^{+}$is the pseudoinverse of matrix $B$.

For this definition of $\delta u(t)$ shown in Equation (6) substituting the expression from Equation (5) in the output of the 
perturbed system gives

$y_{\Delta}(t)=C\{e^{A\left(t-t_{0}\right)} x_{0}+\int_{t_{0}}^{t} e^{A(t-\tau)} B \underbrace{[u(\tau)+\delta u(\tau)]}_{u_{\Delta}(\tau)} d \tau\}$

which is the time-domain response of the nominal system excited by the nominal input plus a time-varying perturbation of it.

A direct result of the two propositions is that both uncertainties and faults can be described as variations of the nominal input as in the following expression

$$
\left\{\begin{array}{l}
\dot{x}(t)=A x(t)+B\left[u(t)+f_{o l}(t)\right] \\
y(t)=C x(t)
\end{array}\right.
$$

or in closed-loop form:

$$
\dot{x}(t)=A_{c l} x(t)+r(t)+f(t)
$$

where $A_{c l}$ is the closed-loop dynamics matrix.

The previous considerations raise the question of how a discrimination can be made between these two different cases if an input variation is detected. Typically, uncertainties tend to be more persistent and they have smaller magnitude than faults in the system. Hence, one method of separating faults from uncertainties is to extract a bound for the latter. Since the uncertainty in the model is not only parametric, but also pertains to the model reduction done while approximating the real system, describing the uncertainties by varying the model parameters would give very conservative bounds. Instead, a different approach is followed.

A typical industrial motor [10] (dynamics and PID controller) in closed-loop is a $5^{\text {th }}$-order system. Its approximation as a $2^{\text {nd }}$-order system suggests a $3^{r d}$-order residual pertaining mostly to the high-frequency dynamics of the original system. Let

$$
G_{r 0}(s)=G_{o r}(s)-G(s)
$$

be the additive residual system, with $G_{o r}(s)$ and $G(s)$ being the original and the approximated system, respectively. By means of grey-box identification, its parameters $\left(a_{r i}, b_{r i}, i=1,2,3\right)$ can be estimated by using a series of recorded input/input perturbations pairs ${ }^{1}$.

In order for the parametric uncertainties to be accounted for, the residual model parameters are varied up to a certain percentage (e.g. $\pm 10 \%$ ), and the different systems obtained are plotted with respect to amplitude in the frequency domain as in Figure 2. A curve (dashed line) that bounds every plot from above is then drawn and the corresponding transfer function $f_{t h r}(s)$ is estimated as shown in [11]. This ensures that all the uncertainties will be smaller in amplitude from the $f_{t h r}(s)$. Hence, the uncertainty in the model that arises if the system is excited by an input signal $r(t)$ can be confined to the zone $\pm f_{\text {thr }}(t)$, where $f_{\text {thr }}(t)=\mathcal{L}^{-1}\left\{f_{\text {thr }}(s) R(s)\right\}$

\footnotetext{
${ }^{1}$ The input perturbations can be estimated in the same way as the faults. This method which is based on the use of a Kalman filter will be presented in the next section.
}

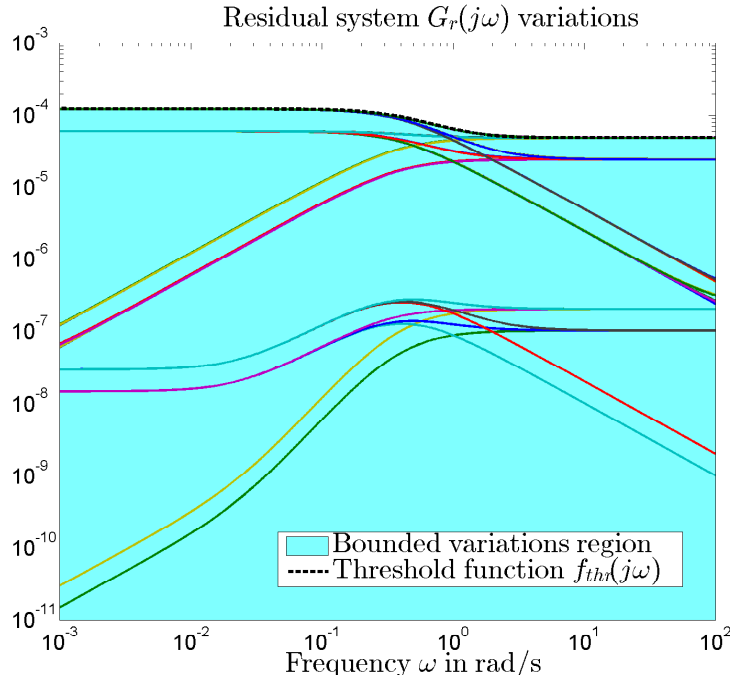

Fig. 2: Bode plot of model variations and uncertainties bound.

and $\mathcal{L}^{-1}$ denotes the inverse Laplace transform operator. As a more direct interpretation of this, $f_{t h r}(t)$ can be viewed as the time response of the residual system $G_{r 0}$ in the worst case (i.e. for the maximum considered uncertainty) when the latter is excited by the same input that excites the real system.

\section{Degradation FAults}

The number of residual generators for a second order linear system

$$
G(s)=\frac{b_{1} s+b_{0}}{s^{2}+a_{1} s+a_{0}}
$$

with $p=1$ outputs and $d=0$ disturbances, even without uncertainties, is $n_{r}=p-d=1$, as described in the left nullspace technique presented in [12]. This implies that a discrimination cannot made between actuator faults and sensor faults in the same motor. That is one of the limitations imposed by the lack of redundant sensors and/or actuators.

\section{A. Fault Diagnosis}

1) Kalman Filter: The diagnostic and estimation method for degradation faults is based on a Kalman filter and a decision policy connected to the uncertainty threshold function $f_{t h r}$ mentioned in the previous section. The additive variation in the input of the system is modelled as a state with constant autonomous behaviour, which is estimated by the filter. The augmented system is written in state-space controller canonical form as

$$
\begin{gathered}
\dot{x}=\underbrace{\left[\begin{array}{cc|c}
0 & 1 & 0 \\
-a_{0} & -a_{1} & 1 \\
\hline 0 & 0 & 0
\end{array}\right]}_{A} x+\underbrace{\left[\begin{array}{l}
0 \\
1 \\
0
\end{array}\right]}_{B} u+B_{v} w \\
y=\underbrace{\left[\begin{array}{cc|c}
b_{0} & b_{1} \mid
\end{array}\right]}_{C} x+v
\end{gathered}
$$

where $\chi$ is the state vector of the approximated system in controller canonical form, $x^{T}(t)=\left[\begin{array}{ll}\chi^{T}(t) & \delta(t)\end{array}\right]$ and $w \sim$ $\mathcal{N}(0, Q)$ and $v \sim \mathcal{N}(0, R)$ are the process and measurement 
noise, respectively, with $Q \geq 0, R>0$. The covariance matrix $Q$ for the Kalman filter is designed with the variance corresponding to $\delta(t)$ being set to a higher value compared to the other two states, so that the filter estimates completely unknown dynamics, so long the latter are slower than the dynamics of the filter itself.

Following the terminology introduced in [13], a fault signature function $\mathcal{H}(t): \mathbb{R} \rightarrow\{0,1\}$ can be defined as

$$
\mathcal{H}(t)=\left\{\begin{array}{l}
0 \text { if }\left|f_{t h r}(t)\right|-|\delta(t)| \geq 0 \text { (nominal) } \\
1 \text { if }\left|f_{\text {thr }}(t)\right|-|\delta(t)|<0 \text { (faulty) }
\end{array}\right.
$$

The inequalities above constitute the Fault Diagnosis Decision Policy for the servomotor.

2) Statistical Change Detection: From the definition of $\mathcal{H}$ and $f_{\text {thr }}$, it is clear that faults with amplitude less than or equal to $\left|f_{t h r}(t)\right|-|\delta(t)|$ cannot be detected. This can be remedied with the use of statistical change detection methods such as the Generalised Likelihood Ratio (GLR) algorithm.

Let $\mathcal{H}_{0}, \mathcal{H}_{1}$ be two hypotheses defined as

$$
\begin{aligned}
& \mathcal{H}_{0}: \delta \sim \mathcal{N}\left(\mu_{0}, \sigma^{2}\right) \\
& \mathcal{H}_{1}: \delta \sim \mathcal{N}\left(\mu_{1}, \sigma^{2}\right)
\end{aligned}
$$

where $\delta$ is the input perturbation estimated by the Kalman filter affected by Gaussian white noise. The GLR test is used to generate a decision as to whether the mean value of $\delta$ changes from $\mu_{0}$ to $\mu_{1}$, the latter being unknown. Note that the variance of $\delta$ is assumed to be the same in both hypotheses.

If $p_{\mu_{0}}(\delta)$ and $p_{\mu_{1}}(\delta)$ are the likelihood functions of $\delta$ (i.e. the probability density evaluated exactly at $\delta$ and depended only on the mean value of the stochastic variable) for the two hypotheses, respectively, then by monitoring a function of the cumulative log-likelihood ratio

$$
S_{j}^{k}\left(\mu_{1}\right)=\sum_{i=j}^{k} \ln \frac{p_{\mu_{1}}(\delta(i))}{p_{\mu_{0}}(\delta(i))}
$$

a choice can be made between these two hypotheses. More specifically, the decision function

$$
g(k)=\max _{k-M+1 \leq j \leq k} \max _{\mu_{1}} S_{j}^{k}\left(\mu_{1}\right)
$$

can be used to replace the fault detection decision policy in (10) with the following:

$$
\left\{\begin{array}{l}
\text { Accept } \mathcal{H}_{0} \text { if } g(k) \leq h \\
\text { Accept } \mathcal{H}_{1} \text { if } g(k)>h
\end{array}\right.
$$

Since no change in the variance of $\delta$ is assumed and since in both hypotheses $\delta$ follows a normal distribution, the decision function $g(k)$ can explicitly be written as ([12]):

$$
g(k)=\frac{1}{2 \sigma^{2}} \max _{k-M+1 \leq j \leq k} \frac{1}{k-j+1}\left[\sum_{i=j}^{k}\left(\delta(i)-\mu_{0}\right)\right]^{2}
$$

In the formulation of (14) both $M$, which is the maximum number of sampling periods that the fault can be detected in and the threshold value $h$, are design parameters that can be chosen after offline tuning, i.e. by selecting the maximum value of $g(k)$ over a set of nominal operation data. Moreover, $\mu_{0}$ is typically very close to 0 since no input perturbations are expected in the nominal case. A block diagram of the fault estimation setup is shown in Figure 3.

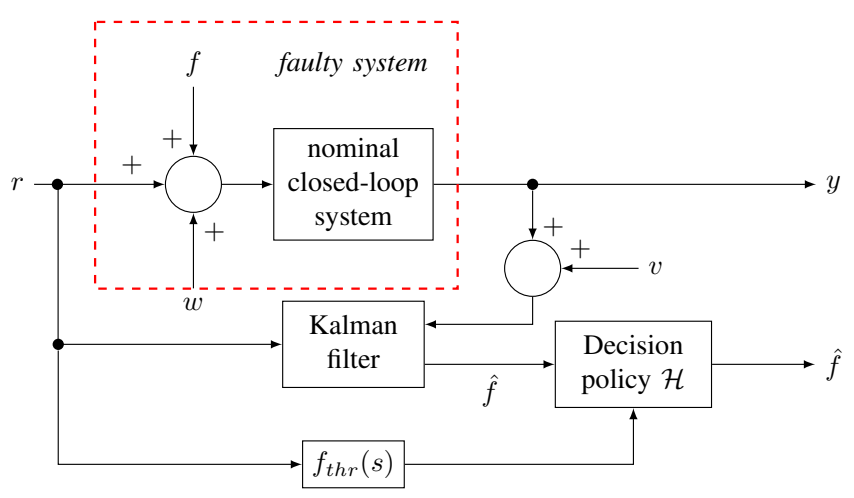

Fig. 3: Fault estimation setup.

\section{B. Fault accommodation}

The remedial actions, in the case that a degradation fault is detected, pertain to the online correction of the reference signal, so that the fault is directly compensated for. The fault correction setup is seen in Figure 4.

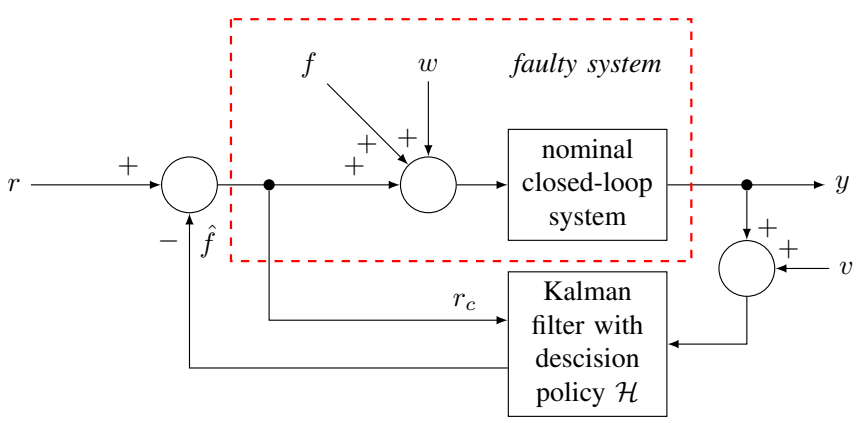

Fig. 4: Reference correction setup.

By comparing the faulty system description in Figure 1(b) with the reference-correction setup in figure 4 , it can be seen that in absence of complete component failures the compensated system has the same I/O behaviour with the nominal system, since in the ideal case $\hat{f} \rightarrow \delta$ and these two terms cancel each other.

The design of the Klaman filter gain matrix $L$ is not affected by the system reference correction. This is a direct result of the Separation Theorem in linear systems ([14]). Indeed, the dynamics of the corrected system and the Kalman filter are written as

$$
\begin{aligned}
& \dot{x}=A x+B r-B \psi \\
& y=C x \\
& \dot{\hat{x}}=(A-L C) \hat{x}+B(r-\psi)+L y \\
& \psi=C_{\delta} \hat{x}
\end{aligned}
$$




$$
\left[\begin{array}{c}
\dot{x} \\
\hat{x}
\end{array}\right]=\left[\begin{array}{c|c}
A & -B C_{\delta} \\
\hline L C & A-L C-B C_{\delta}
\end{array}\right]\left[\begin{array}{l}
x \\
\hat{x}
\end{array}\right]+\left[\begin{array}{c}
B \\
B
\end{array}\right] r
$$

where $\hat{x}$ is the estimated state vector, $\psi=\hat{\delta}$ is the compensation term and $C_{\delta}=\left[\begin{array}{lll}0 & 0 & 1\end{array}\right]$. Then, if $e=x-\hat{x}$ is the estimation error, the augmented system pertaining to $\left[\begin{array}{ll}x^{T} & e^{T}\end{array}\right]^{T}$ is written as

$$
\left[\begin{array}{c}
\dot{x} \\
\dot{e}
\end{array}\right]=\left[\begin{array}{c|c}
A-B C_{\delta} & -B C_{\delta} \\
\hline \mathbf{0}_{3 \times 3} & A-L C
\end{array}\right]\left[\begin{array}{l}
x \\
\hat{e}
\end{array}\right]+\left[\begin{array}{c}
B \\
\mathbf{0}_{3 \times 1}
\end{array}\right] r
$$

Its eigenvalues are the concatenation of the filter's eigenvalues and those of the closed-loop system $\left(A-B C_{\delta}\right)$. Hence, the Kalman filter dynamics can be shaped independently.

Moreover, the characteristic polynomial of $A_{c o r}=A-$ $B C_{\delta}$ is $P_{A_{\text {cor }}}(\lambda)=\lambda P_{A}(\lambda)$ showing that the eigenvalues of the original system are not affected by the compensation. This is desirable since the nominal behaviour of the system should be maintained after accommodation of any faults.

In case of a constant fault, the PID controller will asymptotically reject it as disturbance. This will limit the detection to the transient time. However, the speed of rejection depends on the controller tuning, which may allow performance degradation. Moreover, the proposed black-box FTC scheme, considers a fault only if there is discrepancy between real and expected output. Hence, if the effect of a fault is rejected very fast, then it is not considered as a fault any more.

\section{Sensor faults}

This method treats actuator and sensor degradation faults the same. This is valid from the perspective of estimating and accommodating non-nominal variations in the system. However, since the position encoder is the only sensor that provides the necessary information for the output feedback control, the sensor measurement will still not agree with the actual position of the motor after the correction of the reference due to a sensor fault. This could cause problems to the event-driven dynamics management in an industrial process that includes the motor.

\section{Component failure Detection}

Two separate cases of component failures for the servomotor system will be examined in this section. In the first case, the actuation signal $u$ is zero for all time after a specific time instant $t_{f}$, while in the second case the same happens for the output readings $y$.

$$
\begin{cases}\text { Actuator failure: } & u(t)=0, t \geq t_{f} \\ \text { Sensor failure: } & y(t)=0, t \geq t_{f}\end{cases}
$$

Considering the equivalent descriptions of the faulty system shown in Figure 1, a complete failure of the actuator or the sensor of the motor can be viewed as a persistent (that can be not compensated for with the reference-correction setup) perturbation signal in the reference of the nominal closedloop system. Since the failures are very specific types of faults, the dynamic profile of this reference perturbation can be determined in advance in each of the two cases. Then the idea for detecting the failure is to use statistical methods to examine whether this perturbation that is estimated by the Kalman filter matches the dynamic profile of any of the two specific faults.

\section{A. Actuator failure}

Let $G, K, r, u, u_{f}, y$ and $y_{f}$ be the open-loop transfer function of the nominal system, the transfer function of the controller, the reference, nominal input, faulty input, nominal output and faulty output, respectively, for the system in frequency domain. And let $u_{f}=(1-\Delta) u, 0 \leq \Delta \leq 1$, where $\Delta=0$ corresponds to the fault-free case and $\Delta=1$ to a complete failure of the actuator. Then

$$
\begin{array}{r}
y_{f}=G u_{f}=G(1-\Delta) u=G(1-\Delta) K\left(r-y_{f}\right) \\
(1+G K) y_{f}=G K r-\Delta G K(\underbrace{r-y_{f}}_{e_{f}})=G K r-\Delta G K e_{f} \\
y_{f}=\underbrace{\frac{G K}{1+G K}}_{G_{c l}}\left(r-\Delta \cdot e_{f}\right) \Leftrightarrow y_{f}=G_{c l}\left(r-\Delta \cdot e_{f}\right)
\end{array}
$$

In case of a complete actuator failure, $\Delta=1$ and $y_{f}=$ $G_{c l}\left(r-e_{f}\right)$. In accordance to Figure 1(b) the estimated reference perturbation $\delta$ should match the signal

$$
\varrho=-e_{f}=y_{f}-r
$$

\section{B. Sensor failure}

The same notation as in the case of actuator failures is considered but now with the fault being at the output of the system like in the following expression

$$
y_{f}=(1-\Delta) y, 0 \leq \Delta \leq 1
$$

where $\Delta=0$ corresponds to the fault-free case and $\Delta=1$ to a complete failure of the sensor. Then

$$
\begin{aligned}
& y_{f}=(1-\Delta) y=G_{c l}(1-\Delta) r=G_{c l}(r-\Delta \cdot r) \Rightarrow \\
& y_{f}=G_{c l}(r+\delta)
\end{aligned}
$$

In case of a complete sensor failure, $\Delta=1$ and the estimated reference perturbation $\delta$ should match the signal

$$
\varrho=-r
$$

\section{Detection of dynamical profile matching with CUSUM}

In the same line with the GLR test used for detection of degradation faults, the Cumulative Sum (CUSUM) algorithm is used for testing whether the estimated reference perturbation $\delta$ matches the dynamical profile of a specific known or measurable signal that can be associated to failure of actuator or sensor in the system. Using the results presented in [12] and [15] a decision function $g_{c}(k)$ can be considered for selecting between the following two hypotheses:

$$
\begin{cases}\mathcal{H}_{\text {nom }}: \delta \text { matches } \mu_{0} & \text { if } g_{c}(k) \leq h_{c} \\ \mathcal{H}_{\text {fail }}: \delta \text { matches } \mu_{0}+\varrho(k) & \text { if } g_{c}(k)>h_{c}\end{cases}
$$

where $\mu_{0}$ is the mean value of the reference perturbation, typically very close to zero in the nominal case, and $\varrho(k)$ 
is available at every time instant. The decision function is given by [12]:

$$
\left\{\begin{array}{l}
g_{c}(k)=\max _{1 \leq j \leq k} S_{j}^{k} \\
S_{j}^{k}=\sum_{i=j}^{k} \frac{\varrho(i)}{\sigma^{2}}\left(\delta(i)-\frac{2 \mu_{0}+\varrho(i)}{2}\right)
\end{array}\right.
$$

where $S_{j}^{k}$ is the cumulative sum of the log-likelihood ratio of the two hypotheses.

Hence, for detecting actuator failures $\delta$ is compared to $\mu_{0}+y_{f}-r$, while for sensor failures it is compared to $\mu_{0}-r$. Similarly to the GLR test, the threshold value can be chosen offline, by inspecting the evolution of $g(k)$ over a set of nominal operation data.

In both cases the method should be applied to the system when the fault-compensation mechanism is active. This is necessary so as to exclude the case of large magnitude faults that can otherwise be corrected through the reference, i.e. ascertain that the fault is indeed "persistent", before deciding that the actuator (or sensor) has completely failed. Figures 5 and 6 show the complete FTC setup and the fault-tolerant operation scheme, respectively.

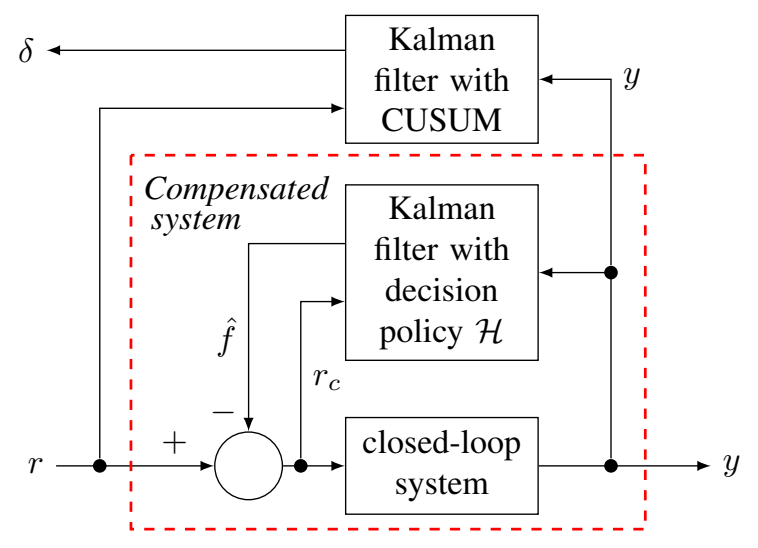

Fig. 5: Complete FTC setup.

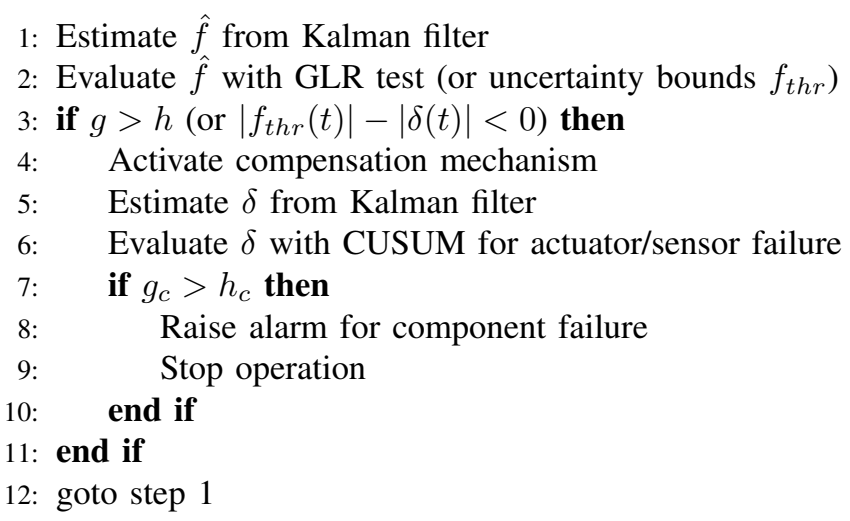

Fig. 6: Fault-tolerant operation scheme

\section{Simulation RESUlts}

The movement of a single-axis robot is simulated for a trajectory from $0 \mathrm{~m}$ to $0.5 \mathrm{~m}$ in $3 \mathrm{~s}$.

\section{A. Additive actuator faults}

A series of step changes in the control signal are considered for the case of degradation faults. For a direct comparison with the estimated reference perturbation $\delta(t)$, the signal that could generate the fault, i.e. $f_{\text {ref }}(t)=$ $\mathcal{L}^{-1}\left\{K_{P I D}^{-1}(s) f(s)\right\}$ is considered. The motor position and
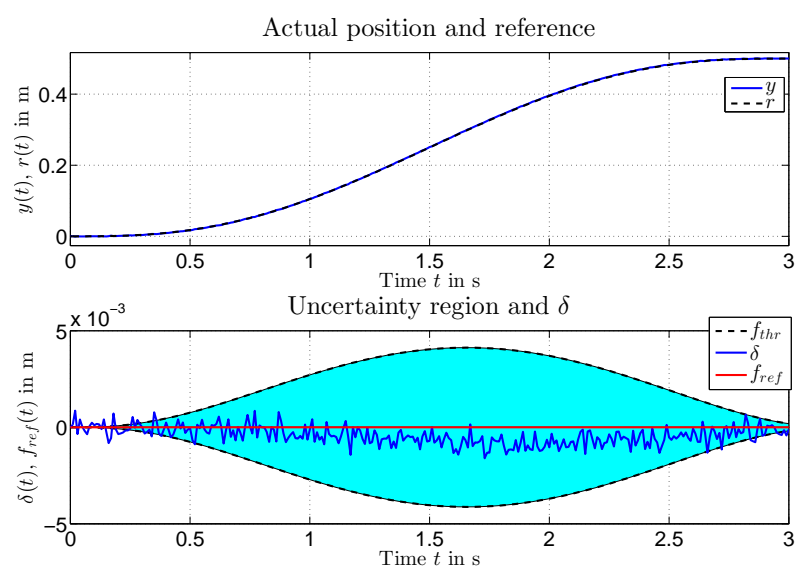

Fig. 7: Nominal operation.

reference signal are shown in Figure 7 (top) in nominal operation. The cyan area in the bottom plot describes the uncertainty region, which encloses the estimated reference perturbation.

In the presence of the considered fault, the estimation grows out of the uncertainty region and compensation for the estimated fault in the reference is then enabled. This can be seen in Figure 8. The deviations from the nominal
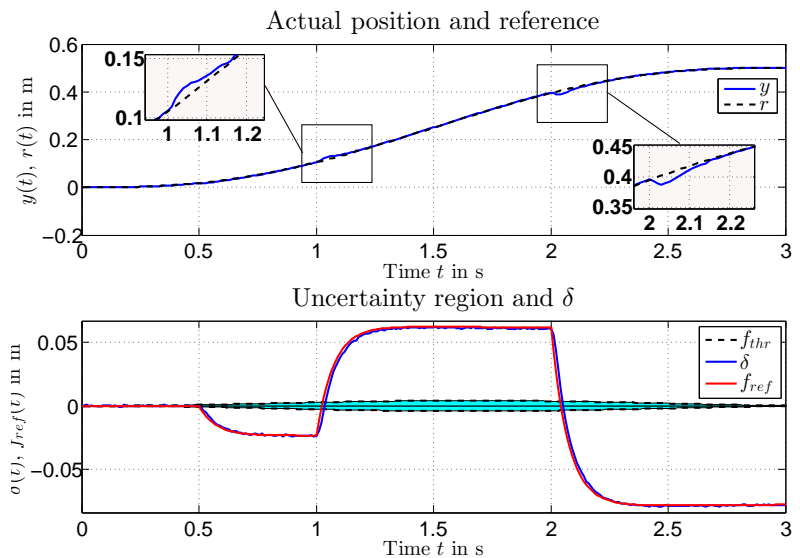

Fig. 8: Degradation additive fault in the actuator.

trajectory are relatively small and are corrected in less than 0.1 sec. This can be also seen from the GLR test shown in Figure 9. The GLR parameters where chosen as $M=25$ samples and $h=17$.

\section{B. Actuator and sensor failure}

To illustrate component failures, two cases are shown bellow. In the first, the actuator fails at $t=1 \mathrm{sec}$, while in the second the sensor fails at the same time instant. Figures 10 and 11 show the performance of the detection method for 

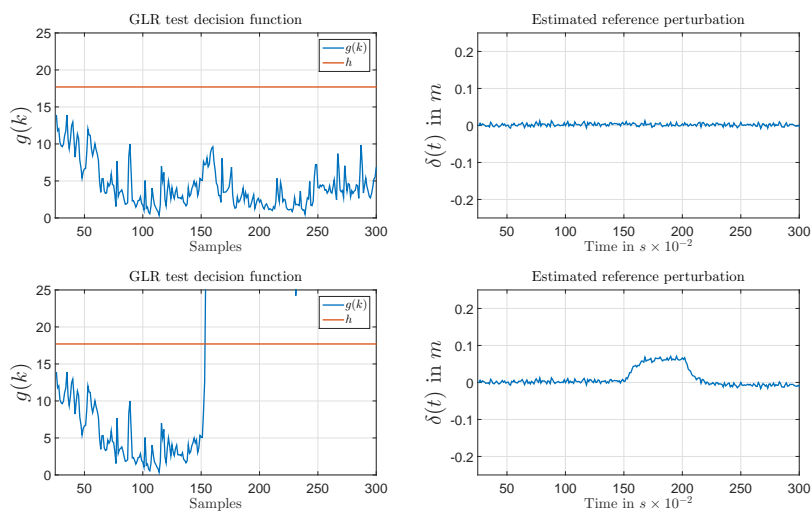

Fig. 9: GLR test for nominal (top) and faulty (bottom) operation.

two cases, respectively, with threshold value $h_{c}=800$. The failures are detected in less than $0.1 \mathrm{~s}$.
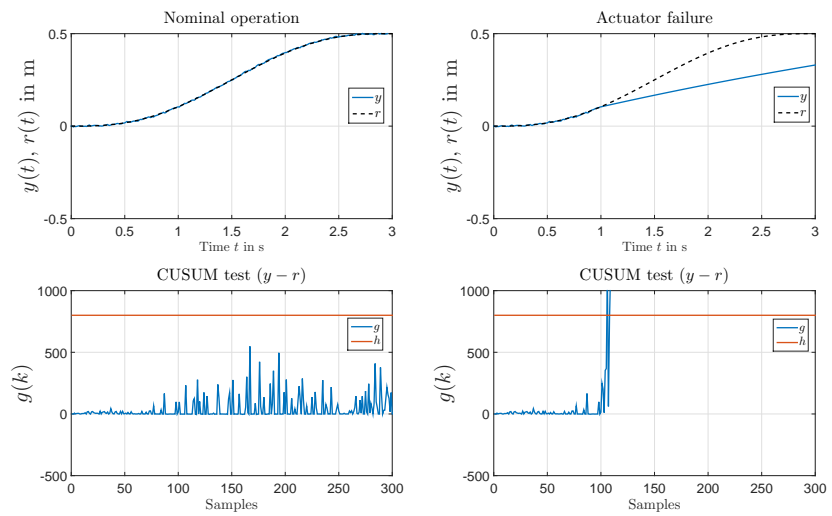

Fig. 10: Nominal operation and actuator failure.
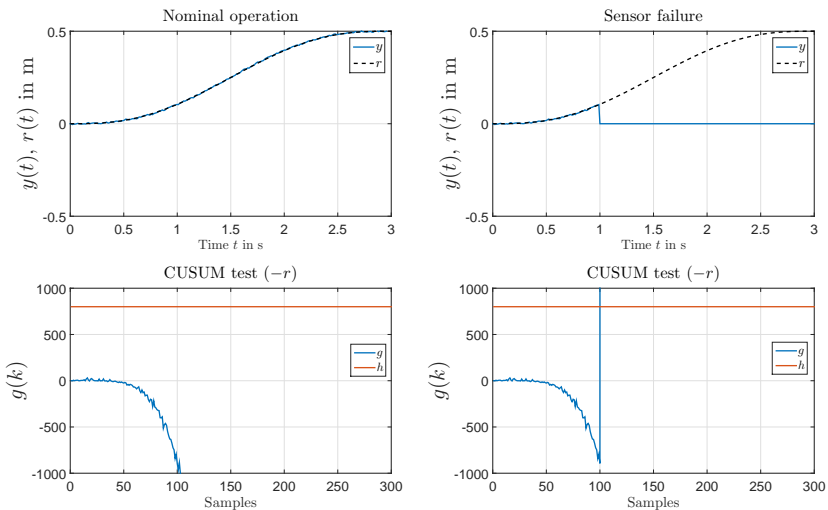

Fig. 11: Nominal operation and sensor failure.

\section{CONCLUSION}

A holistic approach for a generic FTC scheme with limited system knowledge in an industrial servomotor was presented in this paper. A method based on I/O mapping and model uncertainty bounds was used to obtain a model for the servomotor. Moreover, a technique based on the GLR test, the Kalman filter and online reference correction was used to detect, estimate and compensate for degradation faults, respectively. The results showed that the effectiveness of the method relies on the description of the model uncertainty bounds, the selection of the sensitivity threshold in the GLR test and the dynamics of the fault. Lastly, a method for detecting component failures was developed based on statistical CUSUM tests for detecting matchings of the estimated reference perturbation signal to known dynamical profiles. The method was applied to two specific types of failures (zero actuation signal and zero measurements) which can be extended to the more general case of "stuck" actuator/sensor failure. The detection time was fast in comparison to the system dynamics and it primarily depends on the selection of the threshold value in the CUSUM algorithm.

\section{REFERENCES}

[1] A. M. Ali, C. Join, F. Hamelin et al., "Fault diagnosis of uncertain linear system using structural knowledge," 7th IFAC Symposium on Fault Detection, Supervision and Safety of Technical Processes, Safeprocess 2009, 2009.

[2] H. Niemann and N. Kjølstad, "Active Fault Diagnosis for Systems with Reduced Model Information." IFAC Proceedings Volumes (IFACPapersOnline), pp. 965-970, 2009.

[3] M. Laursen, M. Blanke, and D. Düstegör, "Fault diagnosis of a water for injection system using enhanced structural isolation," International Journal of Applied Mathematics and Computer Science, vol. 18, no. 4, pp. 593-603, 2008.

[4] T. Khan, M. Arshad, and M. Choudhry, "Modeling and control of cartesian robot manipulator," in 9th International Multitopic Conference, IEEE INMIC 2005, Dec 2005, pp. 1-4.

[5] M. W. Spong, S. Hutchinson, and M. Vidyasagar, Robot Modeling and Control, 1st ed. Wiley, 112005.

[6] T. C. S. Hsia, T. A. Lasky, and Z. Guo, "Robust independent joint controller design for industrial robot manipulators," IEEE Transactions on Industrial Electronics, vol. 38, no. 1, pp. 21-25, 1991.

[7] S. Skogestad and I. Postlethwaite, Multivariable Feedback Control: Analysis and Design, 2nd ed. Wiley-Interscience, 112005.

[8] K. Zhou, J. C. Doyle, and K. Glover, Robust and Optimal Control, 1st ed. Prentice Hall, 81995.

[9] L. Ljung, System Identification: Theory for the User (2nd Edition), 2nd ed. Prentice Hall, 11999.

[10] M. Blanke, S. Bgh, R. Jrgensen, and R. Paton, "Fault detection for a diesel engine actuator - a benchmark for fdi," Control Engineering Practice, vol. 3, no. 12, pp. 1731-1740, 1995.

[11] K. Zhou and J. C. Doyle, Essentials of Robust Control, 1st ed. Prentice Hall, 101997.

[12] M. Blanke, M. Kinnaert, J. Lunze, and M. Staroswiecki, Diagnosis and Fault-Tolerant Control, 2nd ed. Springer, 82006.

[13] H. Niemann, "Active fault diagnosis in closed-loop uncertain systems," IFAC Proceedings Volumes (IFAC-PapersOnline), vol. 6, no. 1, pp. 587-592, 2006.

[14] B. D. O. Anderson and J. B. Moore, Optimal Control: Linear Quadratic Methods (Dover Books on Engineering). Dover Publications, 22007.

[15] M. Basseville and I. V. Nikiforov, Detection of Abrupt Changes: Theory and Application. Upper Saddle River, NJ, USA: PrenticeHall, Inc., 1993. 